\title{
Growth and Generalized Iterated Functions
}

\author{
Nintu Mandal ${ }^{1}$, Ratan Kumar Dutta ${ }^{2}$ \\ ${ }^{1}$ Department of Mathematics, Chandernagore College, Chandernagore, Hooghly-712136, West Bengal, India \\ ${ }^{2}$ Department of Mathematics, Netaji Mahavidyalaya,Arambagh, Hooghly-712601, West Bengal, India \\ Email:nintu311209@gmail.com,ratan_3128@yahoo.com
}

\begin{abstract}
We study the growth properties of iterated entire functions. Considering entire functions of finite iterated order we prove some results on the growth properties of generalized iterations. The results improve and generalize some earlier results.
\end{abstract}

Keywords-Entire functions, growth, iteration.

\section{INTRODUCTION, DEFINITIONS AND NOTATIONS}

To study the growth properties of generalized iterated entire functions some relevant notations and definitions are needed. For standard definitions and notations we refer to [4].

Notation 1.1 [10] Let $\log ^{[0]} t=t$, $\exp ^{[0]} t=t$ and for positive integer $k, \quad \log ^{[k]} t=\log \left(\log ^{[k-1]} t\right)$, $\exp ^{[k]} t=\exp \left(\exp ^{[k-1]} t\right)$.

Definition 1.2 The order $\rho_{(f)}$ and lower order $\lambda_{(f)}$ of a meromorphic function $f$ is defined as

and

$$
\rho_{(f)}=\limsup _{r \rightarrow \infty} \frac{\log T(r, f)}{\log r}
$$

$$
\lambda_{(f)}=\liminf _{r \rightarrow \infty} \frac{\log T(r, f)}{\log r} .
$$

If $f$ is an entire function, then one can easily verify that

and

$$
\rho_{(f)}=\limsup _{r \rightarrow \infty} \frac{\log ^{[2]} M(r, f)}{\log r}
$$

$$
\lambda_{(f)}=\liminf _{r \rightarrow \infty} \frac{\log ^{[2]} M(r, f)}{\log r} .
$$

Definition 1.3 A function $\lambda_{(f)}(r)$ is called a lower proximate order of a meromorphic function $f$ if

(i) $\quad \lambda_{(f)}(r)$ is nonnegative and continuous for $r \geq r_{0}$, say;

(ii) $\quad \lambda_{(f)}(r)$ is differentiable for $r \geq r_{0}$ except possibly at isolated points at which $\lambda_{(f)}^{\prime}(r-0)$ and $\lambda^{\prime}{ }_{(f)}(r+0)$ exist;

(iii) $\quad \lim _{r \rightarrow \infty} \lambda_{(f)}(r)=\lambda_{(f)}<\infty$;

(iv) $\quad \lim _{r \rightarrow \infty} r \lambda^{\prime}(f)(r) \log r=0$

and

(v) $\quad \lim \inf _{r \rightarrow \infty} \frac{T(r, f)}{r^{\lambda}(f)^{(r)}}=1$.

Definition 1.4 [1] Let $f$ and $g$ be two entire functions defined in the open complex plane and $c \in(0,1]$. Then the generalized iterations of $f$ with respect to $g$ are defined as follows:

$$
\begin{aligned}
& f_{(1, g)}(z)=(1-c) z+c f(z) \\
& f_{(2, g)}(z)=(1-c) g_{(1, f)}(z)+c f\left(g_{(1, f)}(z)\right) \\
& f_{(3, g)}(z)=(1-c) g_{(2, f)}(z)+c f\left(g_{(2, f)}(z)\right) \\
& f_{(n, g)}(z)=(1-c) g_{(n-1, f)}(z)+c f\left(g_{(n-1, f)}(z)\right)
\end{aligned}
$$

and so are

$g_{(1, f)}(z)=(1-c) z+c g(z)$

$g_{(2, f)}(z)=(1-c) f_{(1, g)}(z)+c g\left(f_{(1, g)}(z)\right)$

$g_{(3, f)}(z)=(1-c) f_{(2, g)}(z)+c g\left(f_{(2, g)}(z)\right)$

$g_{(n, f)}(z)=(1-c) f_{(n-1, g)}(z)+c g\left(f_{(n-1, g)}(z)\right)$.

Clearly all $f_{(n, g)}(z)$ and $g_{(n, f)}(z)$ are entire functions.

For two non-constant entire functions $f(z)$ and $g(z)$, it is well known that

$$
\log M(r, f(g)) \leq \log M(M(r, g), f) .
$$

Let $f(z)$ and $g(z)$ be any two transcendental entire functions defined in the open complex plane $\mathbb{C}$. J. Clunie [3] proved that $\lim _{r \rightarrow \infty} \frac{\log T(r, f \circ g)}{T(r, f)}=\infty$ and $\lim _{r \rightarrow \infty} \frac{\log T(r, f \circ g)}{T(r, g)}=0$. In 1985, Singh [11] proved some comparative growth properties of $\log T(r, f$ 。 $g)$ and $T(r, f)$. After this Lahiri [6] proved some results on the comparative growth of $\log T(r, f \circ$ $g)$ and $T(r, g)$.

Recently Lahiri and Datta [7] made a close investigation on the comparative growth properties of $\log T(r, f \circ g)$ and $T(r, g)$. They also proved some results on the comparative growth properties of $\log \log T(r, f \circ g)$ and $T\left(r, f^{(k)}\right)$. In 2011 Banerjee and Dutta [2] proved some results on comparative growth of iterated entire functions which improve some earlier results. 


\section{E-ISSN: 2321-9637}

\section{Available online at www.ijrat.org}

In this paper, we study the growth of generalized iterated entire functions and prove some results which generalize and improve some earlier results.

\section{LEMMAS}

Lemma 2.1 [4] Let $f$ be an entire function. For $0 \leq r<R<\infty$, we have

$$
T(r, f) \leq \log ^{+} M(r, f) \leq \frac{R+r}{R-r} T(R, f) .
$$

Lemma 2.2 [4] Let $f$ and $g$ be two transcendental entire functions. Then

$$
\frac{T(r, f)}{T(r, g(f))} \rightarrow 0 \text { as } r \rightarrow \infty .
$$

Lemma 2.3 [8] Let $f$ and $g$ be two entire functions. If $M(r, g)>\frac{(2+\theta)}{\theta}|g(0)|$ for any $\theta>0$, then

$$
T(r, f(g))<(1+\theta) T(M(r, g), f) .
$$

In particular if $g(0)=0$, then $T(r, f(g)) \leq$ $T(M(r, g), f)$ for all $r>0$.

Lemma 2.4 [9] Let $f$ and $g$ be two entire functions. Then we have

$$
T(r, f(g)) \geq \frac{1}{3} \log M\left(\frac{1}{8} M\left(\frac{r}{4}, g\right)+O(1), f\right) .
$$

Lemma 2.5 [5] Let $f$ be an entire function. Then for $k>2$,

$$
\underset{r \rightarrow \infty}{\liminf } \frac{\log ^{[k-1]} M(r, f)}{\log ^{[k-2]} T(r, f)}=1 .
$$

Lemma 2.6 [7] Let $f$ be a meromorphic function. Then for $\delta(>0)$, the function $r^{\lambda_{(f)}+\delta-\lambda_{(f)}(r)}$ is an increasing function of $r$.

Lemma 2.7 [9] Let $f$ and $g$ be two non-constant entire functions such that $0<\lambda_{(f)} \leq \rho_{(f)}<\infty$ and $0<\lambda_{(g)} \leq \rho_{(g)}<\infty$. Then for any $\theta(0<$ $\left.\theta<\min \left\{\lambda_{(f)}, \lambda_{(g)}\right\}\right)$

$$
\begin{aligned}
& \quad \log ^{[n-1]} T\left(r, f_{(n, g)}\right) \\
& \quad \leq\left(\rho_{(f)}+\theta\right)(1+O(1)) \log M(r, g) \\
& \quad+O(1), \text { when } n \text { is even, } \\
& \quad \log ^{[n-1]} T\left(r, f_{(n, g)}\right) \\
& \quad \leq\left(\rho_{(g)}+\theta\right)(1+O(1)) \log M(r, f) \\
& \quad+O(1), \text { when } n \text { is odd, } \\
& \log ^{[n-1]} T\left(r, f_{(n, g)}\right) \\
& \geq(1+O(1))\left(\lambda_{(f)}-\theta\right) \log M\left(\frac{r}{4^{n-1}}, f\right) \\
& +O(1), \text { when } n \text { is even, }
\end{aligned}
$$

and

$$
\begin{aligned}
& \log ^{[n-1]} T\left(r, f_{(n, g)}\right) \\
& \geq(1+0(1))\left(\lambda_{(g)}-\theta\right) \log M\left(\frac{r}{4^{n-1}}, f\right) \\
& +O(1), \text { when } n \text { is odd }
\end{aligned}
$$

for all large values of $r$.

Proof. We get from Lemma 2.2, Lemma 2.3 and (1) for $\theta(>0)$ and for all large values of $r$,

$$
\begin{aligned}
& T\left(r, f_{(n, g)}\right) \\
& \leq T\left(r, g_{(n-1, f)}\right)+T\left(r, f\left(g_{(n-1, f)}\right)\right)+O(1) \\
& \leq(1+0(1)) T\left(r, f\left(g_{(n-1, f)}\right)\right) \\
& \leq 2 T\left(M\left(r, g_{(n-1, f)}\right), f\right)
\end{aligned}
$$

Hence using Definition 1.1 we have

$$
\begin{aligned}
& \log T\left(r, f_{(n, g)}\right) \\
& \leq \log T\left(M\left(r, g_{(n-1, f)}\right), f\right)+O(1) \\
& \leq\left(\rho_{(f)}+\theta\right) \log M\left(r, g_{(n-1, f)}\right) \\
& +O(1)
\end{aligned}
$$

Hence

$$
\begin{aligned}
& \log ^{[2]} T\left(r, f_{(n, g)}\right) \leq \log ^{[2]} M\left(r, g_{(n-1, f)}\right)+O(1) \\
& \leq \log \left\{\log M\left(r, f_{(n-2, g)}\right)+\log M\left(r, g\left(f_{(n-2, g)}\right)\right)\right. \\
& +O(1)\}+O(1) \\
& \leq \log \left\{\log M\left(M\left(r, f_{(n-2, g)}\right), g\right)\right. \\
& \left.+\log M\left(M\left(r, f_{(n-2, g)}\right), g\right)+O(1)\right\}+O(1) \\
& \leq \log \log M\left(M\left(r, f_{(n-2, g)}\right), g\right)+O(1) \\
& \leq\left(\rho_{(g)}+\theta\right) \log M\left(r, f_{(n-2, g)}\right) \\
& +O(1)
\end{aligned}
$$

Therefore

$$
\begin{aligned}
& \log ^{[n-1]} T\left(r, f_{(n, g)}\right) \\
& \leq\left(\rho_{(f)}+\theta\right) \log M\left(r, g_{(1, f)}\right)+O(1) \\
& \leq\left(\rho_{(f)}+\theta\right)\{\log M(r, z)+\log M(r, g)+O(1)\} \\
& +O(1) \\
& \leq\left(\rho_{(f)}+\theta\right)(1+O(1)) \log M(r, g) \\
& +O(1), \text { when } n \text { is even }
\end{aligned}
$$

Similarly,

$$
\begin{aligned}
& \log ^{[n-1]} T\left(r, f_{(n, g)}\right) \\
& \leq\left(\rho_{(g)}+\theta\right)(1+O(1)) \log M(r, f) \\
& +O(1), \text { when } n \text { is odd }
\end{aligned}
$$

Again for $\theta\left(0<\theta<\min \left\{\lambda_{(f)}, \lambda_{(g)}\right\}\right)$, we get from Lemma 2.2 and Lemma 2.4 for all large values of $r$ 


\section{E-ISSN: 2321-9637}

\section{Available online at www.ijrat.org}

$$
\begin{aligned}
& T\left(r, f_{(n, g)}\right) \\
\geq & T\left(r, f\left(g_{(n-1, f)}\right)\right)-T\left(r, g_{(n-1, f)}\right)+O(1) \\
= & (1+0(1)) T\left(r, f\left(g_{(n-1, f)}\right)\right) \\
\geq & (1+0(1)) \frac{1}{3} \log M\left(\frac{1}{8} M\left(\frac{r}{4}, g_{(n-1, f)}\right)+O(1), f\right) \\
\geq & (1+0(1)) \frac{1}{3}\left[\frac{1}{8} M\left(\frac{r}{4}, g_{(n-1, f)}\right)+O(1)\right]^{\lambda_{(f)}-\theta} \\
\geq & (1+0(1)) \frac{1}{3}\left[\frac{1}{9} M\left(\frac{r}{4}, g_{(n-1, f)}\right)\right]^{\lambda_{(f)}-\theta}
\end{aligned}
$$

So

$$
\begin{aligned}
& \log T\left(r, f_{(n, g)}\right) \\
& \geq\left(\lambda_{(f)}-\theta\right) \log M\left(\frac{r}{4}, g_{(n-1, f)}\right)+O(1) \\
& \geq\left(\lambda_{(f)}-\theta\right) T\left(\frac{r}{4}, g_{(n-1, f)}\right)+O(1) \\
& \geq\left(\lambda_{(f)}-\theta\right)\left[T\left(\frac{r}{4}, g\left(f_{(n-2, g)}\right)\right)-T\left(\frac{r}{4}, f_{(n-2, g)}\right)\right. \\
& +O(1)]+O(1) \\
& \geq\left(\lambda_{(f)}-\theta\right)(1+O(1)) T\left(\frac{r}{4}, g\left(f_{(n-2, g)}\right)\right)+O(1) \\
& \geq\left(\lambda_{(f)}-\theta\right) \frac{1}{3} \log M\left(\frac{1}{8} M\left(\frac{r}{4^{2}}, f_{(n-2, g)}\right)+O(1), g\right) \\
& +O(1) \\
& \geq\left(\lambda_{(f)}-\theta\right) \frac{1}{3}\left[\frac{1}{8} M\left(\frac{r}{4^{2}}, f_{(n-2, g)}\right)+O(1)\right]^{\lambda_{(g)}-\theta} \\
& +O(1) \\
& \geq\left(\lambda_{(f)}-\theta\right) \frac{1}{3}\left[\frac{1}{9} M\left(\frac{r}{4^{2}}, f_{(n-2, g)}\right)\right]^{\lambda_{(g)}-\theta} \\
& +O(1)
\end{aligned}
$$

Therefore

$$
\begin{aligned}
& \log ^{[n-1]} T\left(r, f_{(n, g)}\right) \\
& \geq\left(\lambda_{(f)}-\theta\right) \log M\left(\frac{r}{4^{n-1}}, g_{(1, f)}\right)+O(1) \\
& \geq(1+0(1))\left(\lambda_{(f)}-\theta\right) \log M\left(\frac{r}{4^{n-1}}, g\right) \\
& +O(1), \text { when } n \text { is even. }
\end{aligned}
$$

Similarly

$$
\begin{aligned}
& \log ^{[n-1]} T\left(r, f_{(n, g)}\right) \\
& \geq(1+0(1))\left(\lambda_{(g)}-\theta\right) \log M\left(\frac{r}{4^{n-1}}, f\right) \\
& +O(1), \text { when } n \text { is odd. }
\end{aligned}
$$

This proves the lemma.

\section{THEOREMS}

Theorem 3.1 Let $f(z)$ and $g(z)$ be two entire functions such that $0<\lambda_{(f)} \leq \rho_{(f)}<\infty$ and $0<\lambda_{(g)} \leq \rho_{(g)}<\infty$. Then for $k=0,1,2,3, \ldots \ldots$

(i)

$$
\begin{aligned}
& \frac{\lambda_{(g)}}{\rho_{(f)}} \leq \underset{r \rightarrow \infty}{\liminf } \frac{\log ^{[n]} T\left(r, f_{(n, g)}\right)}{\log T\left(r, f^{(k)}\right)} \\
& \leq \underset{r \rightarrow \infty}{\lim \sup } \frac{\log ^{[n]} T\left(r, f_{(n, g)}\right)}{\log T\left(r, f^{(k)}\right)} \\
& \leq \frac{\rho_{(g)}}{\lambda_{(f)}}, \text { when } n \text { is even. }
\end{aligned}
$$

(ii)

$$
\begin{aligned}
& \frac{\lambda_{(f)}}{\rho_{(g)}} \\
& \leq \underset{r \rightarrow \infty}{\liminf _{(\log } \frac{\log ^{[n]} T\left(r, f_{(n, g)}\right)}{\log T\left(r, g^{(k)}\right)}} \\
& \leq \underset{r \rightarrow \infty}{\operatorname{lom} \sup } \frac{\log ^{[n]} T\left(r, f_{(n, g)}\right)}{\log T\left(r, g^{(k)}\right)} \\
& \leq \frac{\rho_{(f)}}{\lambda_{(g)}}, \text { when } n \text { is odd. }
\end{aligned}
$$

Proof. First suppose that $n$ is even. Then for given $\theta\left(0<\theta<\min \left\{\lambda_{(f)}, \lambda_{(g)}\right\}\right)$ we have from Lemma 2.7 for all large values of $r$,

$$
\begin{aligned}
& \quad \log ^{[n-1]} T\left(r, f_{(n, g)}\right) \\
& \quad \leq\left(\rho_{(f)}+\theta\right)(1+O(1)) \log M(r, g) \\
& \quad+O(1) \\
& \text { i.e. } \log ^{[n]} T\left(r, f_{(n, g)}\right) \leq \log ^{[2]} M(r, g)+O(1)
\end{aligned}
$$

Also we know that

$$
\lim i n f_{r \rightarrow \infty} \frac{\log T\left(r, g^{(k)}\right)}{\log r}=\lambda_{(g)} .
$$

Now

$$
\begin{aligned}
& \limsup _{r \rightarrow \infty} \frac{\log ^{[n]} T\left(r, f_{(n, g)}\right)}{\log T\left(r, f^{(k)}\right)} \leq \underset{r \rightarrow \infty}{\limsup } \frac{\log ^{[2]} M(r, g)}{\log T\left(r, f^{(k)}\right)} \\
& \leq \limsup _{r \rightarrow \infty}\left[\frac{\log ^{[2]} M(r, g)}{\log r} \cdot \frac{\log r}{\log T\left(r, f^{(k)}\right)}\right] \\
& =\frac{\rho_{(g)}}{\lambda_{(f)}}
\end{aligned}
$$

Again from lemma 2.7 we have for all large values of $r$,

$$
\begin{aligned}
& \log ^{[n-1]} T\left(r, f_{(n, g)}\right) \\
& \left.\geq\left(\lambda_{(f)}-\theta\right)(1+O(1)) \log M\left(\frac{r}{4^{n-1}}, g\right)+O(1)\right) \\
& \geq\left(\lambda_{(f)}-\theta\right)(1+O(1))\left(\frac{r}{4^{n-1}}\right)^{\lambda_{(g)}-\theta} \\
& +O(1)
\end{aligned}
$$

i.e. $\log ^{[n]} T\left(r, f_{(n, g)}\right) \geq\left(\lambda_{(g)}-\theta\right) \log r+O(1)$.

Also

Therefore 


\section{Available online at $w w w . i j r a t . o r g$}

$$
\frac{\log ^{[n]} T\left(r, f_{(n, g)}\right)}{\log T\left(r, f^{(k)}\right)} \geq \frac{\left(\lambda_{(g)}-\theta\right) \log r+O(1)}{\left(\rho_{(f)}+\theta\right) \log r}
$$

Since $\theta>0$ is arbitrary we get

$$
\liminf _{r \rightarrow \infty} \frac{\log ^{[n]} T\left(r, f_{(n, g)}\right)}{\log T\left(r, f^{(k)}\right)} \geq \frac{\lambda_{(g)}}{\rho_{(f)}} .
$$

Therefore from (2) and (3) we have the result for even $n$.

Similarly for odd $n$ we have (ii).

This proves the theorem.

Theorem 3.2 Let $f$ and $g$ be two entire functions such that $0<\lambda_{(f)} \leq \rho_{(f)}<\infty$ and $\rho_{(g)}<\infty$. Then for $k=0,1,2,3, \ldots \ldots$

$\lim _{r \rightarrow \infty} \frac{\log ^{[n-1]} T\left(r, f_{(n, g)}\right)}{T\left(\exp (r), f^{(k)}\right)}=0$ for all natural number $n(\geq 2)$.

Proof. First suppose $n$ is even. Then by Lemma 2.7 for all sufficiently large values of $r$ and $\theta(0<$ $\left.\theta<\lambda_{(f)}\right)$

$$
\begin{aligned}
& \log { }^{[n-1]} T\left(r, f_{(n, g)}\right) \\
& \leq\left(\rho_{(f)}+\theta\right)(1+O(1)) \log M(r, g) \\
& +O(1), \\
& \log M(r, g)<r^{\rho_{(g)}+\theta}
\end{aligned}
$$

and $\quad T\left(\exp (r), f^{(k)}\right)>e^{r^{\left(\lambda(f)^{-\theta}\right)}}$

So

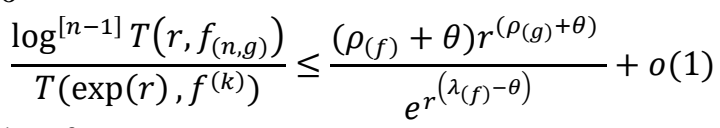

Therefore

$$
\lim _{r \rightarrow \infty} \frac{\log ^{[n-1]} T\left(r, f_{(n, g)}\right)}{T\left(\exp (r), f^{(k)}\right)}=0
$$

Similarly for odd $\mathrm{n}$ we have

$$
\begin{aligned}
& \log ^{[n-1]} T\left(r, f_{(n, g)}\right) \\
& \leq\left(\rho_{(g)}+\theta\right)(1+O(1)) \log M(r, f) \\
& +O(1),
\end{aligned}
$$

So

$$
\log M(r, f)<r^{\left(\rho_{(f)}+\theta\right)}
$$

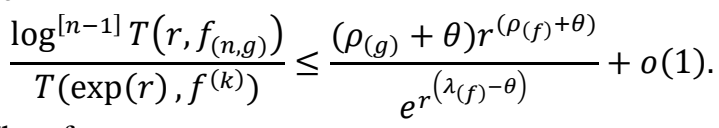

Therefore

This proves the theorem.

$$
\lim _{r \rightarrow \infty} \frac{\log ^{[n-1]} T\left(r, f_{(n, g)}\right)}{T\left(\exp (r), f^{(k)}\right)}=0 .
$$

Remark 3.3 The condition $\rho_{(g)}<\infty$ is necessary for Theorem 3.2 which is shown by the following example.

Example 3.4 Let $f=\exp z, g=\exp ^{[2]} z$ and $c=1$ then

$\lambda_{(f)}=\rho_{(f)}=1$ and $\rho_{(g)}=\infty$.

Now for even $n$
Therefore

$$
f_{(n, g)}=\exp ^{\left[\frac{3 n}{2}\right]} Z
$$

$$
3 T\left(2 r, f_{(n, g)} \geq \log M\left(r, f_{(n, g)}\right)=\exp ^{\left[\frac{3 n}{2}-1\right]} r\right.
$$

i.e. $T\left(r, f_{(n, g)}\right) \geq \frac{1}{3} \exp ^{\left[\frac{3 n}{2}-1\right]} \frac{r}{2}$

Therefore

$$
\begin{aligned}
\log ^{[n-1]} T\left(r, f_{(n, g)}\right) & \geq \exp ^{\left[\frac{3 n}{2}-1-n+1\right]} \frac{r}{2}+o(1) \\
= & \exp ^{\left[\frac{n}{2}\right]} \frac{r}{2}+o(1)
\end{aligned}
$$

Also when $n$ is odd

$$
f_{(n, g)}=\exp ^{\left[\frac{3 n-1}{2}\right]} Z
$$

Therefore

$$
3 T\left(2 r, f_{(n, g)} \geq \log M\left(r, f_{(n, g)}\right)=\exp ^{\left[\frac{3 n-1}{2}-1\right]} r\right.
$$

i.e. $T\left(r, f_{(n, g)}\right) \geq \frac{1}{3} \exp ^{\left[\frac{3 n-1}{2}-1\right]} \frac{r}{2}$

Therefore

$$
\begin{aligned}
\log ^{[n-1]} T\left(r, f_{(n, g)}\right) & \geq \exp ^{\left[\frac{3 n-1}{2}-1-n+1\right]} \frac{r}{2}+o(1) \\
= & \exp ^{\left[\frac{n-1}{2}\right]} \frac{r}{2}+o(1)
\end{aligned}
$$

Also $T\left(\exp (r), f^{(k)}\right)=\frac{e^{r}}{\pi}$.

Therefore when $n$ is even

$$
\begin{aligned}
& \frac{\log ^{[n-1]} T\left(r, f_{(n, g)}\right)}{T\left(\exp (r), f^{(k)}\right)} \geq \frac{\exp ^{\left[\frac{n}{2}\right] \frac{r}{2}+o(1)}}{\frac{e^{r}}{\pi}} \rightarrow \infty \text { as } r \rightarrow \infty \\
& \text { and when } n \text { is odd } \\
& \frac{\log ^{[n-1]} T\left(r, f_{(n, g)}\right)}{T\left(\exp (r), f^{(k)}\right)} \geq \frac{\exp ^{\left[\frac{n-1}{2}\right] \frac{r}{2}+o(1)}}{\frac{e^{r}}{\pi}} \rightarrow \infty \text { as } r \rightarrow \infty .
\end{aligned}
$$

\section{ACKNOWLEDGEMENT}

The Second Author would like to thank the UGC (ERO), India for financial support vide UGC MRP F No. PSW- 040/15-16 (ERO) dated 25th January, 2017.

\section{REFERENCES}

[1] D. Banerjee and N. Mondal, Maximum modulus and maximum term of generalized iterated entire functions, Bulletin of the Allahabad Mathematical Society, 27(1) (2012), 117-131.

[2] D. Banerjee and R. K. Dutta, The growth of iterated entire functions, Bulletin of Mathematical Analysis and Applictions, 3(3), (2011), 35-49

[3] J. Clunie, The composition of entire and meromorphic functions, Mathematical essays dedicated to A. J. Macintyre, Ohio Univ. Press, (1970), 75-92.

[4] W. K. Hayman, Meromorphic Functions, The Clarendon Press, Oxford, 1964.

[5] I. Lahiri, Generalized proximate order of meromorphic functions, Matematnykn Bechnk, 41 (1989), 9-16.

[6] I. Lahiri, Growth of composite integral functions, Indian J. Pure and Appl. Math., 20(9) (1989), 899-907.

[7] I. Lahiri and S. K. Datta, On the growth of composite entire and meromorphic functions, 


\section{Available online at $w w w . i j r a t . o r g$}

Indian J. Pure and Appl. Math., 35(4) (2004), 525-543.

[8] K. Niino and N. Smita, Growth of a composite function of entire functions, Kodai Math. J., 3, (1980), 374-379.

[9] K. Niino and C. C. Yang, Some growth relationships on factors of two composite entire functions, Factorization Theory of Meromorphic Functions and Related Topics, Marcel Dekker Inc. (New York and Basel), (1982), 95-99.

[10]D. Sato, On the rate of growth of entire functions of fast growth, Bull. Amer. Math. Soc., 69 (1963), 411-414.

[11]A. P. Singh, Growth of composite entire functions, Kodai Math. J., 8 (1985), 99-102. 